\title{
Prognostic significance of home blood pressure control on renal and cardiovascular outcomes in elderly patients with chronic kidney disease
}

\author{
Tomonari Okada, Toshiyuki Nakao, Hiroshi Matsumoto, Yume Nagaoka, Ryo Tomaru, Hideaki Iwasawa and \\ Toshikazu Wada
}

The influence of home blood pressure (HBP) control on renal and cardiovascular outcomes is not fully defined, and the optimal blood pressure (BP) target in elderly patients with chronic kidney disease (CKD) remains unknown. To clarify the influence of HBP on the progression of CKD and the occurrence of cardiovascular events in elderly CKD patients, we recruited 104 patients with stage 3 to 5 CKD, who were $\geqslant 70$ years of age. The mean follow-up duration was $39 \pm 15$ months. HBP was measured every morning and evening for 7 consecutive days. HBP data were obtained every 6 months for 79 of these patients. There were significant correlations observed between morning systolic BP (SBP), evening SBP and the change in estimated glomerular filtration rate (eGFR) during the follow-up period (baseline/follow-up; morning $r=-0.55 /-0.51$, evening $r=-0.48 /-0.38$, all $\boldsymbol{P}<0.0001$, baseline: baseline values, follow-up: mean values obtained every 6 months during the follow-up period). Stepwise multivariate regression analysis identified morning SBP and urinary protein excretion as independent predictors of a change in eGFR during the follow-up period. Cox proportional hazards analysis showed that baseline morning SBP, baseline evening SBP and follow-up morning SBP were significantly associated with an increased risk of renal events (hazard ratios; $1.04(95 \% \mathrm{Cl}$, 1.01-1.07), 1.06 (1.02-1.09) and 1.10 (1.04-1.17), respectively). However, Cox proportional hazards analyses showed that there was no significant association between BP and the risk of cardiovascular events. In conclusion, even among elderly CKD patients, HBP is a significant predictor of decline in renal function and the development of end-stage renal disease. In addition, the optimal target BP for elderly CKD patients needs to be clarified.

Hypertension Research (2009) 32, 1123-1129; doi:10.1038/hr.2009.165; published online 9 October 2009

Keywords: cardiovascular disease; chronic kidney disease; elderly patients; home blood pressure

\section{INTRODUCTION}

Japan is rapidly becoming an aged society. The prevalence of chronic kidney disease $(\mathrm{CKD})$ increases as the number of elderly individuals in the population grows, indicating that Japan will see a rise in the prevalence of CKD in the coming years. ${ }^{1}$ The mean age at which patients started chronic dialysis was 67.2 years in 2008 and has tended to increase every year in Japan. ${ }^{2}$ Thus, it is important to prevent the progression of CKD to end-stage renal disease in elderly CKD patients. Hypertension is one of the most important factors associated with the progression of CKD. The current guidelines recommend a target blood pressure (BP) of $<140 / 90 \mathrm{~mm} \mathrm{Hg}$ for elderly hypertensive patients. ${ }^{3,4}$ A further reduction in BP is recommended for CKD patients. Several studies have shown that BP control leads to a reduction in cardiovascular risk among elderly hypertensive patients. ${ }^{5,6}$ However, there are few studies regarding the influence of $\mathrm{BP}$ control on the progression of CKD or cardiovascular risk in elderly CKD patients. Thus, it is not clear whether or not strict BP control provides benefits for elderly CKD patients. In addition, although several studies have shown that home BP (HBP) is more predictive of the progression of CKD than clinic BP, ${ }^{7-10}$ the effect of HBP control on renal outcomes has not been fully evaluated in elderly CKD patients. Therefore, we sought to identify whether or not HBP predicted the progression of $\mathrm{CKD}$ and the occurrence of cardiovascular events in elderly CKD patients.

\section{METHODS}

Patients

We included 104 patients treated at our department between December 2003 and March 2009 who were $\geqslant 70$ years of age and met the eligibility criteria. The inclusion criteria were as follows: (1) patients had to have already been performing HBP measurements at regular intervals before participating in this study; (2) patients had to have been treated for at least 3 months at our clinic; (3) they had to provide a 24 -h urine collection specimen; and (4) their estimated glomerular filtration rates (eGFRs) had to be $<60 \mathrm{ml} \mathrm{min}^{-1}$ per $1.73 \mathrm{~m}^{2}$. We excluded patients who started dialysis therapy or died within a year after study enrollment, patients with malignancies and those whose HBP data 
were judged inaccurate or inadequate. The baseline clinical characteristics of included patients are shown in Table 1 . Underlying renal diseases were diagnosed on the basis of medical history or renal biopsy findings.

\section{BP measurements}

Clinic BP was measured by a nurse using a validated electronic sphygmomanometer (H-55, TERUMO, Tokyo, Japan) with the patients in a seated position after at least 2 minutes of rest between 0900 hours and 1500 hours. A single measurement was recorded. HBP was measured according to the guidelines for HBP measurement of the Japanese Society of Hypertension (JSH). ${ }^{4}$ BP was measured using a semiautomatic arm device with the patients in a seated position after at least 1 minute of rest. The nurses checked to ensure that the patients knew how to use the devices accurately and checked the validity of the devices. BP was measured in the morning, within 1 hour of waking and in the evening just before bedtime. The value of a single measurement was recorded at each of these time points. The patients recorded BP data as described above for 7 days preceding their clinic visit every 6 months. The morning and evening BP values for each patient were calculated as the mean values of the 7 daily measurements at each time point. Samples were collected to test patients' hemoglobin, serum albumin, creatinine and 24-h urinary protein excretion every 6 months. All included patients provided their informed consent regarding the use of their clinical data in this study.

\section{Table 1 Baseline clinical characteristics}

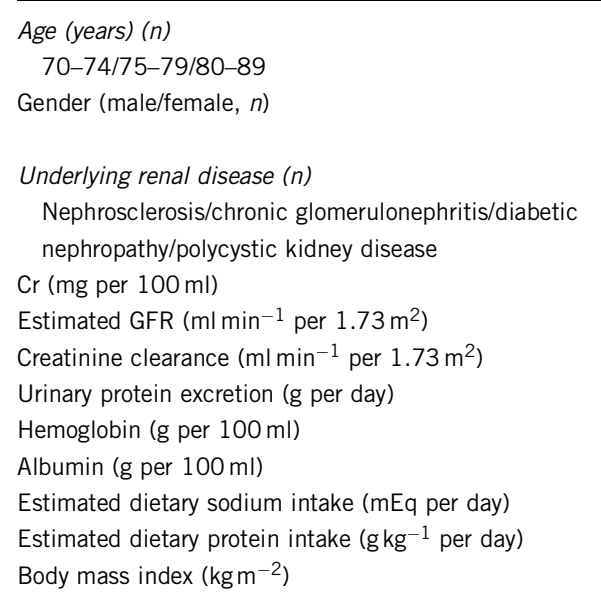

Number of antihypertensive drugs (n) 0/1/2/3 or more

$11 / 27 / 40 / 26$

Class of antihypertensive drugs ( $n$ )

Calcium channel blocker/angiotensin-converting

enzyme inhibitor/angiotensin receptor

blocker/ $\alpha$-blocker/furosemide

Time of administration of antihypertensive drugs

Morning/morning and evening/evening

$41 / 50 / 2$

Blood pressure $(\mathrm{mm} \mathrm{Hg})$
Morning
Evening
Clinic

$142 \pm 18 / 75 \pm 10$

$136 \pm 16 / 70 \pm 9$

$134 \pm 18 / 67 \pm 12$

Patients with a history of cardiovascular events (n) 30 Ischemic heart disease/cerebral infarction/peripheral artery disease/arrhythmia requiring permanent pacemaker implantation/aortic aneurysm

$10 / 10 / 5 / 5 / 5$

\section{Study protocol}

We treated the patients according to the current guidelines for BP control in elderly hypertensive patients. We set up a primary target BP of $<140 /$ $90 \mathrm{~mm} \mathrm{Hg}$ in the clinic and $<135 / 85 \mathrm{~mm} \mathrm{Hg}$ at home. Further reduction of BP was individualized according to each patient's characteristics. Antihypertensive drugs were selected according to the guideline of the JSH. ${ }^{11}$ The mean followup period was $39 \pm 15$ months with a range of 12-65 months. After the baseline examination, the patients visited the clinic every 2-3 months. Of the 104 patients who met the initial inclusion criteria, HBP data and the aforementioned clinical data were obtained every 6 months for 79 patients. Data regarding HBP during the follow-up period were not available for the remaining 25 patients. Follow-up variables were defined as the mean values obtained every 6 months for each of the 79 patients. eGFR was calculated using the following formula: eGFR $=194 \times \mathrm{Cr}^{-1.094} \times$ age $^{-0.287}(\times 0.739$, if female $){ }^{12}$ The annual change in eGFR during the follow-up period was defined by the following formula: ((final eGFR-initial eGFR)/follow-up months $\times 12$ ) $\left(\mathrm{ml} \mathrm{min}{ }^{-1}\right.$ per $1.73 \mathrm{~m}^{2}$ per year). We examined the correlation between the change in eGFR and BP data, and examined the independent variables that influenced the change in eGFR by stepwise multivariate regression analysis. We also examined the influence of BP data on the renal outcomes using KaplanMeier survival curves and multivariate Cox proportional hazards analysis. A renal event was defined as the initiation of chronic dialysis, a doubling of serum creatinine or death.

Acute deterioration of renal function occurred in two patients before the initiation of dialysis due to pneumonia in one patient and heart failure in the other patient. To exclude the influence of these instances of acute deterioration in renal function, the final eGFR was defined as that obtained during the last visit before initiation of dialysis or death. There were no acute changes in renal function observed during the follow-up in any included patients.

In addition, we examined the influence of BP data on cardiovascular outcomes using Kaplan-Meier survival curves and multivariate Cox proportional hazards analyses. Cardiovascular events were defined as death due to cardiovascular causes or new onset of cardiovascular events requiring hospitalization. They included heart failure, ischemic heart disease, arrhythmia requiring permanent pacemaker implantation, cerebral infarction, cerebral hemorrhage and aortic aneurysm requiring surgery. Ischemic heart disease included definite coronary artery disease (diagnosed by coronary angiography), myocardial infarction, percutaneous coronary interventions or coronary artery bypass grafting.

Finally, we evaluated the association between renal and cardiovascular outcomes and HBP measured on two consecutive days (2-day HBP). Data for 2-day HBP was obtained from the mean values of the first and the second day during the 7-day period preceding the clinic visit.

\section{Treatment during the follow-up period}

During the follow-up period, we changed several patients' medication regimens to improve their BP control. The doses or numbers of antihypertensive drugs were increased in 46 patients during the course of the study. They were decreased or changed to another drug in the same class in 21 patients. They were unchanged in 29 patients and were continued not to be administered in 8 patients. Among the 46 patients who received increased doses or additional classes of antihypertensive drugs, 30 received an angiotensin-converting enzyme inhibitor or an angiotensin receptor blocker, 18 received a calcium channel blocker, 23 received an $\alpha$ blocker and 17 received a furosemide. Recombinant human erythropoietin was administered to 46 patients during the follow-up period.

Dietary protein restriction was instructed from dietitian in 45 patients, and the prescribed amount of dietary protein was $0.69 \pm 0.07(0.6-0.8) \mathrm{g} \mathrm{kg}^{-1}$ per day.

Five patients had their drug dosages decreased because of symptomatic orthostatic hypotension. No serious adverse events related to antihypertensive drugs were reported during the follow-up period.

\section{Statistics}

The data were expressed as means \pm s.d. $P$-values of $<0.05$ were considered to be statistically significant. The changes in HBP profiles were analyzed using the 
Wilcoxon signed rank test. Correlations between the change in eGFR and clinical variables were analyzed by Pearson's correlation coefficient and stepwise multivariate regression analysis. Renal and cardiovascular outcomes were assessed using Kaplan-Meier survival analysis and multivariate Cox proportional hazards analysis. Statistical analyses were performed using the Statview computer program (Abacus Concepts, Berkeley, CA, USA).

\section{RESULTS}

Correlations between baseline BP parameters and change in eGFR Figure 1 shows the correlations between baseline morning systolic BP (SBP), evening SBP, clinic SBP and the change in eGFR that occurred during the follow-up period. There were significant correlations observed between all BP values and the change in eGFR. The correlation coefficient between morning SBP and eGFR change was the highest of the various time points tested.

Correlations between baseline variables, follow-up variables and the change in eGFR

Table 2 shows the correlations between baseline variables, follow-up variables and the change in eGFR. The baseline variables and the mean decline rate in eGFR were not significantly different between the 79 patients whose follow-up HBP measurements were available and the remaining 25 patients whose follow-up HBP measurements were not available. Baseline and follow-up SBPs, urinary protein excretion levels and hemoglobin levels were significantly correlated with the change in eGFR that occurred during the follow-up period. The stepwise multivariate regression analysis shown in Table 2 showed that morning SBP and urinary protein excretion, in both the baseline and follow-up data, were significant independent variables associated with the change in eGFR that was observed during the study.

Two-day morning SBP (both the baseline and follow-up values) were also found to be significantly associated with the change in eGFR that occurred during the follow-up period on stepwise multivariate regression analysis $(\beta=-0.41,-0.34)$.

The mean decline in eGFR in patients with diabetic nephropathy was significantly greater than that in patients with other renal diseases $\left(-3.83 \pm 2.41\right.$ versus $-2.18 \pm 3.06 \mathrm{ml} \mathrm{min}^{-1}$ per $1.73 \mathrm{~m}^{2}$ per year, $P=0.006)$. However, all baseline morning SBP, evening SBP and urinary protein excretion values were significantly greater in patients with diabetic nephropathy than in patients with other renal diseases.

Between CKD 4 and 5 patients, the mean decline in eGFR in patients receiving recombinant human erythropoietin $(n=38)$ was significantly greater than that in patients not receiving recombinant human
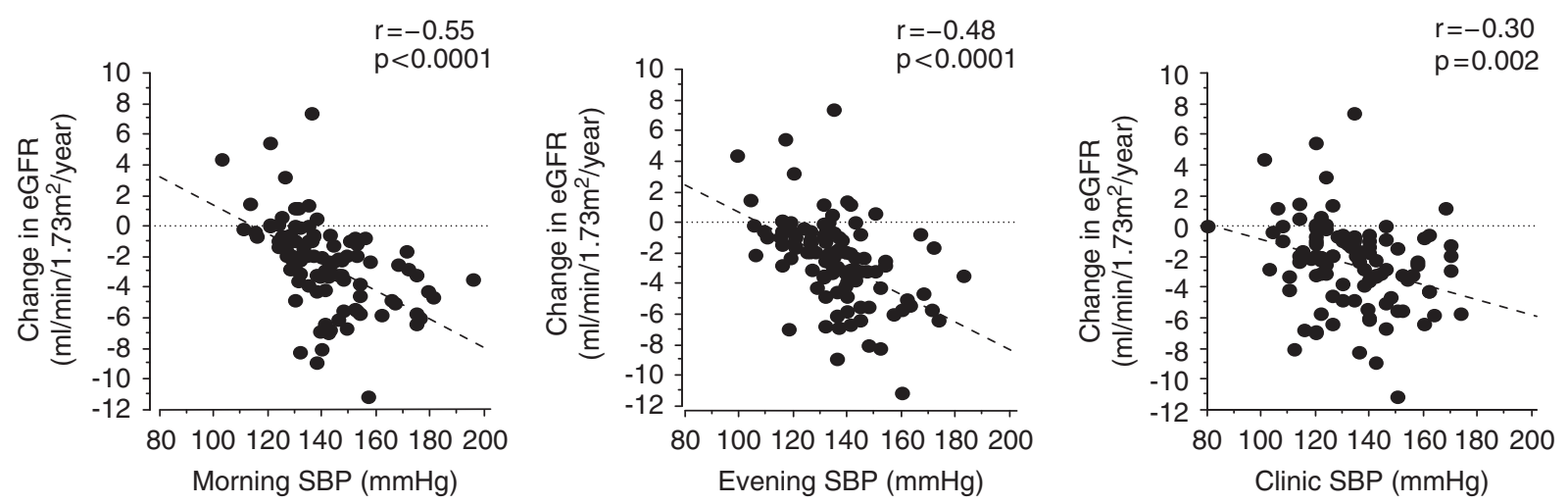

Figure 1 Correlation between baseline morning systolic blood pressure (SBP), evening SBP, clinic SBP and changes in estimated glomerular filtration rate (eGFR).

Table 2 Correlations between baseline clinical variables, follow-up clinical variables and change in eGFR

\begin{tabular}{|c|c|c|c|c|c|c|}
\hline & \multicolumn{3}{|c|}{ Baseline clinical variables } & \multicolumn{3}{|c|}{ Follow-up clinical variables } \\
\hline & \multicolumn{2}{|c|}{ Simple correlations } & $\begin{array}{c}\text { Stepwise multiple regression } \\
\beta\end{array}$ & \multicolumn{2}{|c|}{ Simple correlations } & $\begin{array}{c}\text { Stepwise multiple regression } \\
\beta\end{array}$ \\
\hline Morning SBP & -0.55 & $<0.0001$ & -0.48 & -0.51 & $<0.0001$ & -0.41 \\
\hline Evening SBP & -0.48 & $<0.0001$ & & -0.38 & $<0.0001$ & \\
\hline Evening DBP & -0.14 & 0.15 & & 0.113 & 0.27 & \\
\hline Clinic DBP & -0.04 & 0.71 & & 0.1 & 0.39 & \\
\hline Urinary protein excretion & -0.39 & $<0.0001$ & -0.21 & -0.39 & $<0.0001$ & -0.24 \\
\hline eGFR & 0.09 & 0.93 & & $-0.002^{\mathrm{a}}$ & 0.95 & \\
\hline Hemoglobin & 0.21 & 0.03 & & 0.27 & 0.01 & \\
\hline Serum albumin & 0.15 & 0.13 & & 0.12 & 0.28 & \\
\hline
\end{tabular}

Abbreviations: DBP, diastolic blood pressure; eGFR, estimated glomerular filtration rate; SBP, systolic blood pressure.

aThis means the correlation with baseline eGFR. 
erythropoietin $(n=18)\left(-3.16 \pm 2.20\right.$ versus $-0.94 \pm 3.33 \mathrm{ml} \mathrm{min}^{-1}$ per $1.73 \mathrm{~m}^{2}$ per year, $P=0.008$ ). However, the baseline eGFR was significantly lower and all baseline morning SBPs, evening SBPs and urinary protein excretion levels were significantly greater in patients receiving recombinant human erythropoietin than in those not receiving recombinant human erythropoietin.

There were no significant differences observed in the change in eGFR between patients receiving renin-angiotensin system inhibitors and those not receiving these drugs.

There were no significant differences observed in the change in eGFR among different age subgroups $(<75,75-79$ and $\geqslant 80$ years).

\section{BP profile during the follow-up period}

Among the 79 patients in whom follow-up HBP data could be obtained, the mean baseline morning $\mathrm{BP}$ and evening $\mathrm{BP}$ were $141 \pm 18 / 75 \pm 10$ and $136 \pm 15 / 71 \pm 9 \mathrm{~mm} \mathrm{Hg}$, respectively. The mean final morning BP and evening BP were $138 \pm 14 / 72 \pm 10$ and $134 \pm 13$ / $68 \pm 10 \mathrm{~mm} \mathrm{Hg}$, respectively. Only morning diastolic BP was found to be significantly decreased from baseline $(P<0.01)$. Among the 58 patients in whom HBP data could be obtained 2 years after the baseline BP reading was taken, both the mean morning BP (134 \pm 12 / $71 \pm 9 \mathrm{~mm} \mathrm{Hg})$ and evening BP $(129 \pm 14 / 67 \pm 10 \mathrm{~mm} \mathrm{Hg})$ were found to be significantly decreased as compared with the baseline BP measurement $(P<0.01$ for both). A total of $43 \%$ of the patients $(n=34)$ and $18 \%$ of the patients $(n=14)$ achieved mean morning SBP of $<135 \mathrm{~mm} \mathrm{Hg}$ and $125 \mathrm{~mm} \mathrm{Hg}$, respectively. A total of $58 \%$ of the patients $(n=46)$ and $25 \%$ of the patients $(n=20)$ achieved mean evening SBP of $<135 \mathrm{~mm} \mathrm{Hg}$ and $125 \mathrm{~mm} \mathrm{Hg}$, respectively.

\section{Urinary protein excretion profiles during the follow-up period}

Overall, urinary protein excretion did not significantly change during the follow-up period (final: $1.15 \pm 1.31 \mathrm{~g}$ per day). However, urinary protein excretion did significantly decrease in the 39 patients whose baseline levels were $>1 \mathrm{~g}$ per day (baseline: $2.40 \pm 1.24$, final: $1.91 \pm 1.67 \mathrm{~g}$ per day, $P=0.01)$. The proportion of patients receiving renin-angiotensin system inhibitors was significantly higher in those with a baseline urinary protein excretion of $>1 \mathrm{~g}$ per day as compared with those with a baseline urinary protein excretion of $<1 \mathrm{~g}$ per day ( 82 versus $58 \%, P=0.01$ by $\chi^{2}$-test).

\section{Association between renal events and SBP}

Of the 104 patients included in the study, chronic dialysis was started in 19 patients and a doubling of serum creatinine values occurred in 7 patients. A total of four patients died, and the causes of death were heart failure in one patient, myocardial infarction in one patient and pneumonia in two patients.

Figure 2 shows the Kaplan-Meier survival curves of renal events based on baseline SBP. A baseline morning SBP and a baseline evening SBP of $<135 \mathrm{~mm} \mathrm{Hg}$ was found to be associated with a significantly reduced risk of renal events.

Table 3 shows the hazard ratios (HR) for renal events by SBP categories. After excluding SBP, age, baseline eGFR and baseline urinary protein excretion were found to be the significantly adjusted variables associated with the risk of renal events (age: $\mathrm{HR}=1.16$ (95\% CI, 1.05-1.28; $P<0.01)$; baseline eGFR: HR=0.87 (0.81-0.92; $P<0.001)$; and baseline urinary protein excretion: $H R=1.94(1.43-$ $2.65 ; P<0.001)$ ). These three variables remained significant even after the baseline SBP variable was added back into the Cox models.

Both baseline morning SBP and evening SBP were significantly associated with renal events. A baseline morning SBP of $<135 \mathrm{~mm} \mathrm{Hg}$ indicated a significantly reduced risk of renal events, whereas a baseline morning SBP of $<125 \mathrm{mmHg}$ did not. Both a baseline evening SBP of $<135 \mathrm{mmHg}$ and a baseline evening SBP of $<125 \mathrm{~mm} \mathrm{Hg}$ indicated a significantly reduced risk of renal events. Clinic SBP did not affect renal outcome.

In terms of follow-up BP, morning SBP was significantly associated with the risk of renal events. A morning SBP of $<135 \mathrm{~mm} \mathrm{Hg}$ tended to reduce the risk of renal events.

The coefficients of variation for the baseline morning and evening SBP were $5.6 \pm 2.5 \%$ and $6.2 \pm 3.4 \%$, respectively. Neither the s.d. values nor the coefficient of variation values for home SBP significantly affected renal outcome in either the univariate or multivariate Cox proportional hazards analyses.

The Cox models that included 2-day HBP as independent variable showed that baseline morning and evening 2-day SBP were also significantly associated with renal outcome (2-day morning SBP: $\mathrm{HR}=1.03(95 \% \mathrm{CI}, 1.00-1.06 ; P=0.037)$ and 2-day evening SBP: $\mathrm{HR}=1.05(1.02-1.08, P=0.001))$. Follow-up morning and evening 2-day HBP were also significantly associated with renal outcome (2-day morning SBP: $\mathrm{HR}=1.06$ (95\% CI, 1.02-1.10; $P=0.007)$ and 2-day evening SBP: $\mathrm{HR}=1.07(1.02-1.13, P=0.009))$.

Association between cardiovascular events and SBP Among the 104 patients, a total of 15 cardiovascular events occurred in 12 patients, including 7 incidents of heart failure, 4 ischemic heart disease events, 2 arrhythmias requiring permanent pacemaker implantation, 1 cerebral infarction and 1 cerebral hemorrhage.
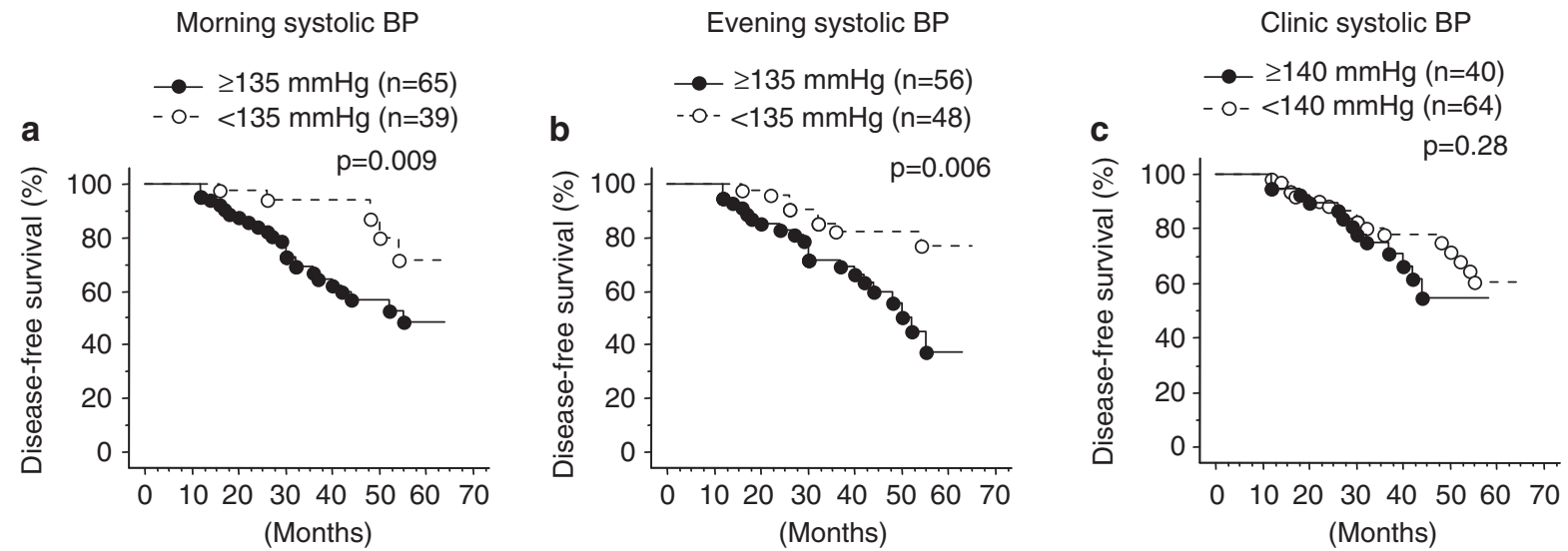

Figure 2 Kaplan-Meier curves for renal events according to baseline systolic blood pressure (BP). 
Table 3 Hazard ratios of renal outcome by SBP

\begin{tabular}{|c|c|c|c|c|}
\hline & \multicolumn{2}{|c|}{ Baseline } & \multicolumn{2}{|c|}{ Follow-up } \\
\hline & Hazard ratio $(95 \% \mathrm{Cl})$ & P-value & Hazard ratio $(95 \% \mathrm{Cl})$ & P-value \\
\hline Evening SBP (per 1 mm Hg) & $1.06(1.02-1.09)$ & 0.0007 & $1.05(1.00-1.11)$ & 0.07 \\
\hline Clinic SBP (per $1 \mathrm{~mm} \mathrm{Hg}$ ) & $1.02(0.99-1.06)$ & 0.17 & $1.04(0.98-1.09)$ & 0.18 \\
\hline Morning SBP $\geqslant 135 \mathrm{~mm} \mathrm{Hg}$ (versus $<135 \mathrm{~mm} \mathrm{Hg}$ ) & $4.90(1.60-15.01)$ & 0.005 & $2.61(0.86-7.93)$ & 0.09 \\
\hline Evening SBP $\geqslant 125 \mathrm{~mm} \mathrm{Hg}$ (versus $<125 \mathrm{~mm} \mathrm{Hg}$ ) & $4.14(1.14-15.01)$ & 0.03 & $2.52(0.63-10.14)$ & 0.19 \\
\hline Clinic SBP $\geqslant 140 \mathrm{~mm} \mathrm{Hg}$ (versus $<140 \mathrm{~mm} \mathrm{Hg}$ ) & $2.62(0.97-7.09)$ & 0.06 & $1.73(0.48-6.24)$ & 0.4 \\
\hline Clinic SBP $\geqslant 130 \mathrm{~mm} \mathrm{Hg}$ (versus $<130 \mathrm{~mm} \mathrm{Hg}$ ) & $1.52(0.56-4.13)$ & 0.41 & $4.31(1.19-15.62)$ & 0.03 \\
\hline
\end{tabular}

Abbreviations: $\mathrm{Cl}$, confidence interval; eGFR, estimated glomerular filtration rate; SBP, systolic blood pressure.

Each SBP variable was adjusted for sex, age, baseline eGFR, urinary protein excretion (UP), hemoglobin (Hb) and serum albumin (Alb). Baseline SBP was adjusted for the each baseline data of UP, $\mathrm{Hb}$ and Alb. Follow-up SBP was adjusted for the each mean value of UP, $\mathrm{Hb}$ and Alb during follow-up.

Morning systolic BP

$\rightarrow \geq 135 \mathrm{mmHg}(\mathrm{n}=65)$

- o- $<135 \mathrm{mmHg}(\mathrm{n}=39)$

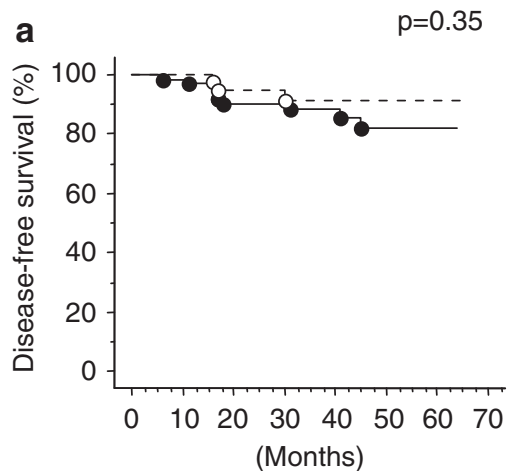

Evening systolic BP

$-\geq 135 \mathrm{mmHg}(\mathrm{n}=56)$

- $0-<135 \mathrm{mmHg}(\mathrm{n}=48)$
Clinic systolic BP

$-\geq 140 \mathrm{mmHg}(\mathrm{n}=40)$

- o- $<140 \mathrm{mmHg}(\mathrm{n}=64)$
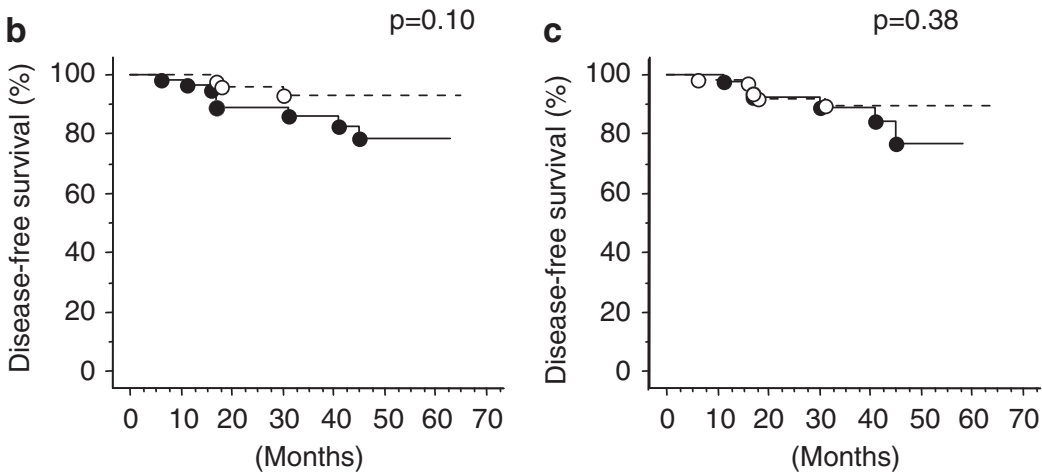

Figure 3 Kaplan-Meier curves for cardiovascular events according to baseline systolic blood pressure (BP).

Table 4 Hazard ratios of cardiovascular outcome by SBP

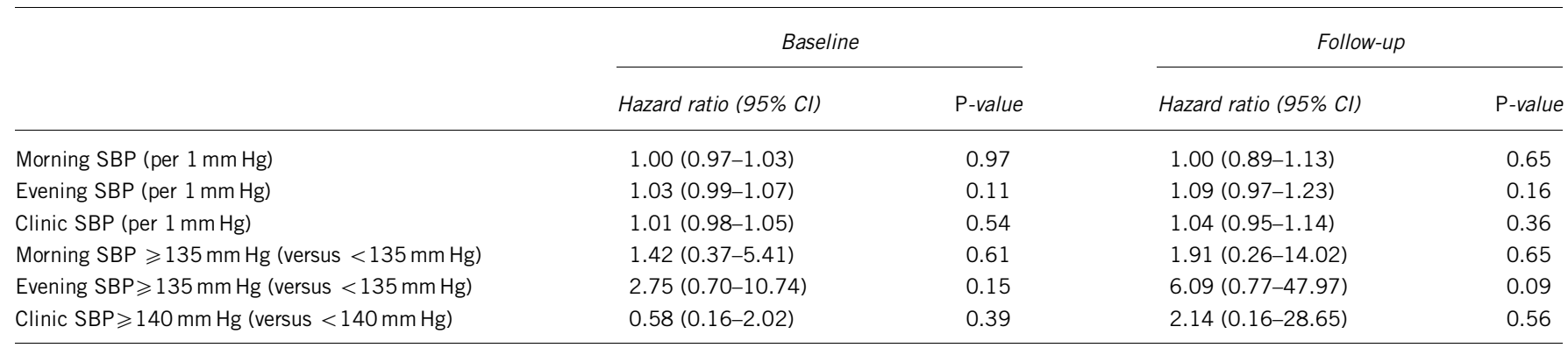

Abbreviations: $\mathrm{Cl}$, confidence interval; eGFR, estimated glomerular filtration rate; SBP, systolic blood pressure.

Each SBP variable was adjusted for sex, age, history of cardiovascular events, baseline eGFR, hemoglobin (Hb) and serum albumin (Alb). Baseline SBP was adjusted for the each baseline data of $\mathrm{Hb}$ and Alb. Follow-up SBP was adjusted for the each mean value of $\mathrm{Hb}$ and Alb during follow-up.

No cardiovascular events developed in any patient whose morning SBP or evening SBP was $<125 \mathrm{~mm} \mathrm{Hg}$ at baseline and during the follow-up. Figure 3 shows the Kaplan-Meier survival analysis examining cardiovascular outcomes according to baseline SBP values. There was no significant difference in outcome noted between the group with a baseline SBP of $<135 \mathrm{~mm} \mathrm{Hg}$ and the group with a baseline SBP of $>135 \mathrm{~mm} \mathrm{Hg}$.

Table 4 shows the HR for cardiovascular events by SBP. Excluding SBP, a history of cardiovascular events was the only significant variable found to affect cardiovascular outcome (HR, 4.25 (95\% CI, 1.24- 
14.59; $P<0.05)$ ). A history of cardiovascular events remained a significant predictor of cardiovascular outcome even when each SBP variable was added to the Cox models. Table 4 shows that each SBP variable was not a significant risk factor for the development of a cardiovascular event. However, increased evening SBP tended to be associated with an increased risk of a cardiovascular event, although it was not statistically significant.

Cox proportional hazards analysis using 2-day HBP as independent variable showed that 2-day HBP was not associated with cardiovascular outcome (data not shown).

\section{Correlations between baseline HBP and clinic BP}

Morning SBP and evening SBP were found to be significantly correlated with clinic SBP $(r=0.42, \quad P=0.0001$ and $r=0.34$, $P=0.0005$, respectively). The correlation between 2-day HBP and clinic BP was also found to be significant. However, the correlation coefficients for 2-day HBP were lower than those of HBP obtained over a 7-day period (2-day morning SBP: $r=0.37, P=0.0001$; 2-day evening SBP: $r=0.28, P=0.005)$.

\section{DISCUSSION}

We examined the prognostic significance of home SBP on the decline in renal function or risk of end-stage renal disease in the elderly CKD patients. These results are consistent with those of the previous studies performed on CKD patients whose age was younger than those in the present study. ${ }^{7-10}$ Masked hypertension is prevalent in CKD patients, ${ }^{13,14}$ and older age is associated with a greater prevalence of masked hypertension among treated hypertensive patients. ${ }^{15,16}$ Thus, HBP measurement might contribute to improved BP control in elderly CKD patients.

It is possible that multiple measurement of HBP might contribute to the superiority of its predictive value. However, the present study showed that 2-day home SBP was also significantly associated with renal outcome. One previous study has also examined the predictive value of 2-day HBP for a diagnosis of CKD among the general population. ${ }^{17}$

Previous studies have shown the influence of BP on the risk of cardiovascular events ${ }^{18-20}$ and the benefit of BP control in lowering cardiovascular risk among elderly hypertensive patients. ${ }^{5,6,21}$ However, only small proportions of CKD patients were included in these studies, and data on renal outcome are scarce in elderly CKD patients. In the Japanese Trial to Assess Optimal Systolic Blood Pressure in Elderly Hypertensive Patients (JATOS), there was no significant difference noted in the incidence of renal failure between the strict treatment group (mean final $\mathrm{BP}, 135.9 / 74.8 \mathrm{~mm} \mathrm{Hg}$ ) and the mild treatment group (mean final BP, $145.6 / 78.1 \mathrm{~mm} \mathrm{Hg}$ ). ${ }^{22}$ In addition, no previous interventional studies have achieved a mean SBP of $<130 \mathrm{~mm} \mathrm{Hg}$. Thus, at present, there is no evidence that a target BP of $<130$ / $80 \mathrm{~mm} \mathrm{Hg}$ is beneficial in terms of prevention of renal events in elderly CKD patients.

The JSH proposed a provisional target HBP of $<135 / 85 \mathrm{~mm} \mathrm{Hg}$ for elderly persons and one of $<125 / 75 \mathrm{~mm} \mathrm{Hg}$ for CKD patients in 2009. ${ }^{4}$ On the basis of the above BP targets, we examined the HR for a home SBP of $>135 \mathrm{~mm} \mathrm{Hg}$ as well as a home SBP of $>125 \mathrm{~mm} \mathrm{Hg}$. We found that baseline morning or evening SBPs of $>135 \mathrm{~mm} \mathrm{Hg}$ significantly predicted an increased risk of renal events, whereas only a baseline evening SBP of $>125 \mathrm{~mm} \mathrm{Hg}$ predicted an increased risk of renal events. Neither a follow-up home SBP of $>135 \mathrm{~mm} \mathrm{Hg}$ nor a follow-up home SBP of $>125 \mathrm{~mm} \mathrm{Hg}$ was associated with an increased risk of renal events. In the present study, we were unable to identify an appropriate target HBP for elderly CKD patients that could potentially reduce the risk of renal events. The validity of the provisional target HBP for CKD patients proposed by JSH should be verified in future studies, and further studies are necessary to determine the most appropriate target BP for preventing renal events in elderly CKD patients.

Chronic kidney disease is an important risk factor for cardiovascular disease. Several previous studies have shown that CKD increases cardiovascular risk among elderly hypertensive patients. ${ }^{23,24}$ However, the association between BP control and cardiovascular risk has not been clearly defined in elderly CKD patients. The present study did not show a significant association between BP control and cardiovascular risk. We propose several possible reasons for these results. First, a history of cardiovascular events might greatly affect the occurrence of future cardiovascular events, independent of BP control. Second, both HBP and clinic BP were better controlled in the present study than in previous studies. For example, the Hypertension in the Very Elderly Trial (HYVET) showed a significant reduction in cardiovascular events in the active-treatment group. ${ }^{6}$ However, the mean achieved BP in the active-treatment group and the placebo group were $143.5 / 77.9 \mathrm{~mm} \mathrm{Hg}$ and $158.5 / 84.0 \mathrm{~mm} \mathrm{Hg}$, respectively. The mean value of final home SBP in the present study was almost the same as the mean value of final SBP in the strict treatment group in JATOS. ${ }^{22}$ Thus, having a well-controlled BP might attenuate the deleterious effect of hypertension on cardiovascular risk in the present study.

The present study produced two interesting findings regarding the possible association between BP control and cardiovascular risk. First, an increase in home evening SBP tended to be associated with an increased cardiovascular risk in the present study. We cannot explain why evening BP was more closely associated with detection of a cardiovascular risk than with morning BP. The Ohasama study found that evening HBP, as well as morning HBP, had a strong predictive significance for risk of stroke. ${ }^{25}$ Second, patients with SBPs of $<125 \mathrm{~mm} \mathrm{Hg}$ had no cardiovascular events in the present study. However, it is not clear whether or not maintaining a home SBP of $<125 \mathrm{~mm} \mathrm{Hg}$ has a beneficial effect on cardiovascular risk in CKD patients. Several studies have shown that lower SBP of $<120 \mathrm{~mm} \mathrm{Hg}$ has an increased risk of cardiovascular events in CKD patients. ${ }^{26,27} \mathrm{On}$ the other hand, The Perindopril Protection Against Recurrent Stroke Study (PROGRESS) has found no association between lowering the SBP target to below $120 \mathrm{~mm} \mathrm{Hg}$ and the risk of stroke in CKD patients. ${ }^{28}$ In the Candesartan Antihypertensive Survival Evaluation in Japan (CASE-J) trial, the risk of cardiovascular events was significantly higher among CKD patients who achieved an SBP of $<130 \mathrm{~mm} \mathrm{Hg}$ than those without CKD who achieved the same level of SBP. ${ }^{29}$ In addition, the risk of cardiovascular events was increased in the CKD patients who achieved an SBP of 130-139 mm Hg compared with those who achieved an SBP of $<130 \mathrm{~mm} \mathrm{Hg}$. These results suggest that strict $\mathrm{BP}$ control might contribute to a reduction of cardiovascular risk in CKD patients.

This study has several limitations. First, the study cohort was small and the duration of the follow-up was relatively short. Thus, the statistical power of this study might be limited, especially for detection of cardiovascular risk. Second, we could not exclude the hemodynamic effects of antihypertensive therapy on the change in eGFR in patients whose antihypertensive drugs were increased during the follow-up period. Third, the ratio of patients over 80 years old at baseline was $22 \%$. Thus, the distribution of age among subjects might be inappropriate to determine the effect of age in elderly people. Fourth, the adjustments we used for the multivariate Cox proportional hazards analysis might have been insufficient. For example, data 
concerning smoking habits was not available. However, we included the main important variables in the Cox analysis, and the influence of other clinical variables on the results might be small.

In conclusion, even among elderly CKD patients, HBP is a significant predictor of decline in renal function and the development of end-stage renal disease. Patients with an SBP of $<125 \mathrm{~mm} \mathrm{Hg}$ had no cardiovascular events in this study, and an increase in home evening SBP tended to be associated with an increased risk of cardiovascular events. Further studies are needed to clarify the optimal target BP (both at home and in the clinic) to prevent the progression of $\mathrm{CKD}$ and to reduce cardiovascular risk in elderly CKD patients.

\section{CONFLICT OF INTEREST}

The authors declare no conflict of interest.

\section{ACKNOWLEDGEMENTS}

We are indebted to Professor J Patrick Barron of the Department of International Medical Communications Center of Tokyo Medical University for his review of this paper.

1 Imai E, Horio M, Iseki K, Yamagata K, Watanabe T, Hara S, Ura N, Kiyohara Y, Hirakata $H$, Moriyama $T$, Ando $Y$, Nitta $K$, Inaguma D, Narita I, Iso $H$, Wakai $K$, Yasuda $Y$, Tsukamoto $\mathrm{Y}$, Ito S, Makino H, Hishida A, Matsuo S. Prevalence of chronic kidney disease (CKD) in the Japanese general population predicted by the MDRD equation modified by a Japanese coefficient. Clin Exp Nephrol 2007; 11: 156-163.

2 Japanese Society for Dialysis Therapy. An overview of regular dialysis treatment in Japan as of 31 December 2008 (in Japanese).

3 Chobanian AV, Bakris GL, Black HR, Cushman WC, Green LA, Izzo Jr JL, Jones DW, Materson BJ, Oparil S, Wright JT, Roccella EJ, the National High Blood Pressure Education Program Coordinating Committee. The Seventh Report of the Joint National Committee on Prevention, Detection, Evaluation, and Treatment of High Blood Pressure: the JNC 7 report. JAMA 2003; 21: 2560-2572.

4 Ogihara T, Kikuchi K, Matsuoka H, Fujita T, Higaki J, Horiuchi M, Imai Y, Imaizumi T, Ito S, Iwao H, Kario K, Kawano Y, Kim-Mitsuyama S, Kimura G, Matsubara H, Matsuura H, Naruse M, Saito I, Shimada K, Shimamoto K, Suzuki H, Takishita S, Tanahashi N, Tsuchihashi T, Uchiyama M, Ueda S, Ueshima H, Umemura S, Ishimitsu T, Rakugi H, on behalf of The Japanese Society of Hypertension Committee for Guidelines for the management of Hypertension. Management and clinical evaluation of blood pressure. Hypertens Res 2009; 32: 11-23.

5 Ogihara T, Nakao K, Fukui T, Fukiyama K, Fujimoto A, Ueshima K, Oba K, Shimamoto K, Matsuoka H, Saruta T, for the CASE-J Trial Group. The optimal target blood pressure for antihypertensive treatment in Japanese elderly patients with high-risk hypertension: a sub-analysis of the Candesartan Antihypertensive Survival Evaluation in Japan (CASEJ) Trial. Hypertens Res 2008; 31: 1595-1601.

6 Beckett NS, Peter R, Fletcher AE, Staessen JA, Liu L, Dumitrascu D, Stoyanovsky V, Antikainen RL, Nikitin Y, Anderson C, Belhani A, Forette F, Rajkumar C, Thijs L, Banya W, Bulpitt CJ, for the HYVET Study Group. Treatment of hypertension in patients 80 years of age or older. N Engl J Med 2008; 358: 1887-1898.

7 Rave K, Bender R, Heise T, Sawicki PT. Value of blood pressure self-monitoring as a predictor of progression of diabetic nephropathy. J Hypertens 1999; 17: 597-601.

8 Suzuki H, Nakamoto H, Okada H, Sugahara S, Kanno Y. Self-measured systolic blood pressure in the morning is a strong indicator of decline of renal function in hypertensive patients with non-diabetic chronic renal insufficiency. Clin Exp Hypertens 2002; 24: 249-260.

9 Agarwal R, Andersen MJ. Prognostic importance of clinic and home blood pressure recordings in patients with chronic kidney disease. Kidney Int 2006; 69: 406-411.

10 Okada T, Nakao T, Matsumoto H, Nagaoka Y. Value of morning home blood pressure as a predictor of decline in renal function patients with chronic kidney disease. $\mathrm{Am} \mathrm{J}$ Nephrol 2008; 28: 982-989.
11 Ogihara T, Kikuchi K, Matsuoka H, Fujita T, Higaki J, Horiuchi M, Imai Y, Imaizumi T, Ito S, Iwao H, Kario K, Kawano Y, Kim-Mitsuyama S, Kimura G, Matsubara H, Matsuura H, Naruse M, Saito I, Shimada K, Shimamoto K, Suzuki H, Takishita S, Tanahashi N, Tsuchihashi T, Uchiyama M, Ueda S, Ueshima H, Umemura S, Ishimitsu T, Rakugi H, on behalf of The Japanese Society of Hypertension Committee for Guidelines for the management of Hypertension. Treatment with antihypertensive drugs. Hypertens Res 2009; 32: 33-39.

12 Matsuo S, Imai E, Horio M, Yasuda Y, Tomita K, Nitta K, Yamagata K, Tomino Y, Yokoyama $\mathrm{H}$, Hishida A, Collaborators developing the Japanese equation for estimated GFR. Revised equations for estimated GFR from serum creatinine in Japan. Am J Kidney Dis 2009; 53: 982-992.

13 Okada T, Nakao T, Matsumoto H, Nagaoka Y. The significance of home blood pressure measurement in patients with chronic kidney diseases. JMAJ 2006; 49: 296-304.

14 Bangash F, Agarwal R. Masked hypertension and white-coat hypertension in chronic kidney disease: a meta-analysis. Clin J Am Soc Nephrol 2009; 4: 656-664.

15 Horikawa T, Obara T, Ohkubo T, Asayama K, Metoki H, Inoue R, Kikuya M, Hashimoto J, Totsune K, Imai Y, the J-HOME Study Group. Difference between home and office blood pressures among treated hypertensive patients from the Japan Home versus Office Blood Pressure Measurement Evaluation (J-HOME) Study. Hypertens Res 2008; 31: 1115-1123.

16 Kabutoya T, Ishikawa J, Hohide S, Eguchi K, Ishikawa S, Shimada K, Kario K. Determinants of negative white-coat effect in treated hypertensive patients: The Jichi Morning Hypertension Research (J-MORE) Study. Am J Hypertens 2009; 22: 35-40.

17 Terawaki H, Metoki H, Nakayama M, Ohkubo T, Kikuya M, Asayama K, Inoue R, Hoshi $\mathrm{H}$, Ito S, Imai Y. Masked hypertension determined by self-measured blood pressure at home and chronic kidney disease in the Japanese general population: The Ohasama study. Hypertens Res 2008; 31: 2129-2135.

18 Bobrie G, Chatellier G, Genes N, Clerson P, Vaur L, Vaisse B, Menard Mallion JM. Cardiovascular prognosis of "masked hypertension" detected by home blood pressure self-measurement in elderly treated hypertensive patients. JAMA 2004; 291: 1342-1349.

19 Fagard RH, Broeke CVD, De Cort P. Prognostic significance of blood pressure measured in the office, at home and during ambulatory monitoring in older patients in general practice. J Hum Hypertens 2005; 19: 801-807.

20 Kario K, Ishikawa J, Picering TG, Hoshide S, Eguchi K, Morinari M, Hoshide Y, Kuroda T, Shimada K. Morning hypertension: the strongest independent risk factor for stroke in elderly hypertensive patients. Hypertens Res 2006; 29: 581-587.

21 Gueyffier F, Bulpitt C, Boissel JP, Schron E, Ekbom T, Fagard R, Casiglia E, Kerlikowske K, Coope J, for the INDANA Group. Antihypertensive drugs in very old people: a subgroup meta-analysis of randomized controlled trials. Lancet 1999; 353: 793-796.

22 JATOS Study Group. Principal results of the Japanese Trial to Assess Optimal Systolic Blood Pressure in Elderly Hypertensive Patients (JATOS). Hypertens Res 2008; 31: 2115-2127.

23 Kagiyama S, Matsumura K, Ansai T, Soh I, Takata Y, Awano S, Sonoki K, Yoshida A, Takehara T, lida M. Chronic kidney disease increases cardiovascular mortality in 80year-old subjects in Japan. Hypertens Res 2008; 31: 2053-2058.

24 Ishikawa J, Shimizu M, Hoshide S, Eguchi K, Pickering TG, Shimada K, Kario K. Cardiovascular risk of dipping status and chronic kidney disease in elderly Japanese hypertensive patients. J Clin Hypertens 2008; 10: 787-794.

25 Asayama K, Ohkubo T, Hara A, Hirose T, Yasui D, Obara T, Metoki H, Inoue R, Kikuya M, Totsune K, Hoshi H, Satoh H, Imai Y. Repeated evening home blood pressure measurement improves prognostic significance for stroke: a 12-year follow-up of the Ohasama study. Blood Press Monit 2009; 14: 93-98.

26 Weiner DE, Tighiouart H, Levey AS, Elsayed E, Griffith JL, Salem DN, Sarnak MJ. Lowest systolic blood pressure is associated with stroke in stage 3 to 4 chronic kidney disease. J Am Soc Nephrol 2007; 18: 960-966.

27 Agarwal R. Blood pressure components and the risk for end-stage renal disease and death in chronic kidney disease. Clin J Am Soc Nephrol 2009; 4: 830-837.

28 Ninomiya T, Perkovic V, Gallagher M, Jardine M, Cass A, Arima H, Anderson C, Neal B, Woodward M, Omae T, MacMahon S, Chalmers J, for the PROGRESS Collaborative Group. Low blood pressure and risk of recurrent stroke in patients with chronic kidney disease: PROGRESS trial. Kidney Int 2008; 73: 963-970.

29 Ogihara T, Saruta T, Rakugi H, Fujimoto A, Ueshima K, Yasuno S, Oba K, Takeda K, Higaki J, Nakao K, on behalf of the CASE-J trial Group. Relationship between the achieved blood pressure and the incidence of cardiovascular events in Japanese hypertensive patients with complications: a sub-analysis of the CASE-J trial. Hypertens Res 2009; 32: 248-254. 